\title{
Crónica
}

\section{CONGRESO CHILENO DE GINECOLOGÍA Y OBSTETRICIA}

\section{Estimados (as) Colegas:}

Me es muy grato invitarlos a participar en el XXXI Congreso de Ginecología y Obstetricia que llevaremos a cabo entre el 20 al 24 de noviembre, en la hermosa ciudad de Viña del Mar (Informaciones: www.sochog.cl/congreso2007). Durante los cuatro días que se desarrollará el encuentro, referentes de la especialidad tanto nacionales como extranjeros, abordarán en diferentes módulos las siguientes temáticas: anticoncepción, piso pélvico, ginecología infanto-juvenil, medicina materno-fetal y reproductiva, oncología ginecológica, sexualidad y terapia de reemplazo hormonal.

Este año estamos muy orgullosos de contar con la presencia de la Presidenta de la Federación Internacional de Ginecología y Obstetricia, Dra. Dorothy Shaw, la primera mujer presidente de FIGO y destacada académica de la University of British Columbia, Vancouver, Canadá.

Junto con esta especial participación, en esta oportunidad también queremos compartir el fruto de nuestra alianza con la Sociedad Canadiense de la especialidad (SOGC), invitándolos a que se inscriban en el Curso de Capacitación Pre Congreso: Advances in Labour and Risk Management (ALARM) que estará dirigido por el Vicepresidente Ejecutivo de la SOGC, Dr. André Lalonde.

ALARM es un programa internacional que tiene como objetivo entregar información sobre las principales causas de la mortalidad materna y neonatal y que además proporciona los lineamientos para gestionar acciones que contribuyen a dis- minuir los lamentables índices de salud materna, perinatal e infantil en países de menores recursos, contribuyendo de esta manera a incrementar las expectativas de vida de las naciones. ALARM se ha realizado con éxito en más de 20 países, convocando a más de mil profesionales y por ello, estamos seguros que la experiencia chilena será igual de satisfactoria.

La invitación está abierta para que participen con nosotros en este encuentro que ha sido organizado con el mejor de los espíritus, el que esperamos se transmita a ustedes para que continuemos mejorando la calidad de la especialidad, con la convicción de estar entregando un pequeño, pero significativo aporte al bienestar y desarrollo del país.

A su vez, nos hemos preocupado de contar con infraestructura y servicios adecuados para garantizar a los asistentes comodidad necesaria, para que cada jornada sea aprovechada al máximo. Asimismo, la planificación del Congreso, ha sido pensada no solamente como una oportunidad para adquirir conocimiento, sino que también como un espacio para compartir y establecer lazos, elemento que siempre es importante para potenciar la unión de los colegas a favor de la actividad médica.

Los espero, reciban un cordial saludo,

Dr. EGHON GuZMÁn B.

Presidente de la Sociedad Chilena de Obstetricia y Ginecología. 


\section{DR. JOHN BILLINGS}

El 2 de abril de 2007, fallece en su hogar a los 89 años tras una breve enfermedad, el médico neurólogo australiano John Billings (1). Junto a su esposa Evelyn fueron los pioneros del desarrollo del método de planificación natural de la familia que lleva su nombre: método de la ovulación o método de Billings.

En 1972, reportan las características de los cambios del moco cervical durante el ciclo menstrual (2) y su asociación con la fertilidad de la mujer. Estos cambios son fácilmente reconocidos por la mujer previamente capacitada y puede utilizarlos tanto para la prevención como la búsqueda de gestaciones. Lo más importante de este método es que no interfiere en la fisiología del proceso reproductivo, por lo que está libre de efectos secundarios, no tiene contraindicaciones médicas, ni costo económico para la usuaria una vez instruida.

Estas características determinaron que la Organización Mundial de la Salud (WHO), desarrollara un estudio multicéntrico (Auckland, Bangalore, Dublín, Manila, El Salvador) para verificar su aplicabilidad y efectividad, los que fueron publicados en la prestigiosa revista Fertility \& Sterility (3-7). Los excelentes resultados de efectividad obtenidos se presentan en la Tabla I (5). Se demostró así que mujeres con fertilidad probada, de diferentes nacio- nalidades y condición socio-cultural, pueden reconocer y diferenciar sus períodos de infertilidad y fertilidad con gran efectividad y utilizarlo en la planificación de sus familias. Posteriormente, WHO lo incluye dentro de las alternativas de métodos para planificación familiar, siendo incorporado en más de 100 países, incluida China donde es el único método de planificación natural de la familia con aprobación gubernamental.

Pérez y cols (8), introdujeron el método en Chile y sus primeros resultados destacan la importancia y las diferencias de la enseñanza por instructores expertos e inexpertos. Actualmente en Chile, hay varios centros privados, públicos y universitarios, donde se capacitan instructoras y usuarias con excelentes resultados, destacando la Unidad de Planificación Natural de la Familia del Servicio de Ginecología y Obstetricia del Hospital Sótero del Río.

Lamentamos profundamente el fallecimiento de este destacado médico, fiel seguidor de los principios hipocráticos del "primum non nocere", por su gran contribución a la familia y la medicina reproductiva.

Dr. ENRIQUE DONOSO S.

Editor Jefe.

Revista Chilena de

Obstetricia y Ginecología.

Tabla I

PROBABILIDAD DE EMBARAZO POR FASE DEL CICLO Y DIAS DESDE EL ULTIMO DÍA DE MOCO CERVICAL TIPO FÉRTIL (DÍA PEACK)

\begin{tabular}{llccc}
\hline Fase del ciclo & $\begin{array}{c}\text { Relación con } \\
\text { el día peak (DP) }\end{array}$ & Ciclos con coito & No de embarazos & $\begin{array}{c}\text { Probabilidad de } \\
\text { embarazo }\end{array}$ \\
\hline Moco pegajoso (sticky) & DP -4 o antes & 81 & 2 & 0,024 \\
Moco pegajoso (sticky) & DP -3 a DP -1 & 6 & 3 & 0,500 \\
Moco resbaloso (slippery) & DP -4 o antes & 17 & 6 & 0,353 \\
Moco resbaloso (slippery) & DP -3 a DP -1 & 22 & 12 & 0,546 \\
Día peak & DP 0 & 9 & 6 & 0,667 \\
Post peak & DP -1 & 18 & 9 & 0,444 \\
Post peak & DP -2 & 44 & 10 & 0,205 \\
Post peak & DP -3 & 112 & 22 & 0,089 \\
Fuera del período fértil & - & 6.158 & 78 & 0,004 \\
Todas las fases & - & 6.467 & & 0,012 \\
\hline
\end{tabular}

Fuente: datos obtenidos de cita 5. 


\section{BIBLIOGRAFÍA}

1. WOOMB. Death of $\mathrm{Dr}$ John Billings. Hallado en: www.woomb.org

2. Billings EL, Billings JJ, Brown JB, Burger JC. Symptom and hormonal changes accompanying ovulation. Lancet 1972;1(7745):282-4.

3. WHO. A prospective multicentre trial of the ovulation method of natural family planning. I. The teaching phase. Fertil Steril 1981;36:152-8.

4. WHO. A prospective multicentre trial of the ovulation method of natural family planning. II. The effectiveness phase. Fertil Steril 1981;36:591-8.
5. WHO. A prospective multicentre trial of the ovulation method of natural family planning. III. Characteristics of the menstrual cycle and of the fertile phase. Fertil Steril 1983;40:773-8.

6. WHO. A prospective multicentre trial of the ovulation method of natural family planning. IV. The outcome of pregnancy. Fertil Steril 1984;41:593-8.

7. WHO. A prospective multicentre trial of the ovulation method of natural family planning. V. Psychosexual aspects. Fertil Steril 1987;47:765-72.

8. Pérez A, Zabala A, Larraín A, et al. The clinical efficiency of the ovulation method (Billings). Rev Chil Obstet Ginecol 1983;48:97-102.

\section{LEY N 20.166 (http://www.mintrab.cl/descargar/ley_20166.pdf)}

La Ley $\mathrm{N}^{\circ} 20.166$, del 12 de febrero de 2007, extiende el derecho de las madres trabajadoras a amamantar a sus hijos aún cuando no exista sala cuna, modificando el artículo 203 y sustituyendo el artículo 206, del Título II del Código del Trabajo: DE LA PROTECCIÓN A LA MATERNIDAD (http:// www.dt.gob.cl/legislacion/1611/printer-59096.html).

Artículo 206. Las trabajadoras tendrán derecho a disponer, a lo menos, de una hora al día, para dar alimento a sus hijos menores de 2 años. Este derecho podrá ejercerse de alguna de las siguientes formas a acordar con el empleador:

a) En cualquier momento dentro de la jornada de trabajo.

b) Dividiéndolo, a solicitud de la interesada, en dos porciones.

c) Postergando o adelantando en media hora, o en una hora, el inicio o el término de la jornada de trabajo.

Este derecho podrá ser ejercido preferentemente en la sala cuna, o en el lugar que se encuentre el menor.
Para todos los efectos legales, el tiempo utilizado se considerará como trabajado.

El derecho a alimentar consagrado en el inciso primero, no podrá ser renunciado en forma alguna y le será aplicable a toda trabajadora que tenga hijos menores de dos años, aun cuando no goce del derecho a sala cuna, según lo preceptuado en el artículo 203.

Tratándose de empresas que estén obligadas a lo preceptuado en el artículo 203, el período de tiempo a que se refiere el inciso primero se ampliará al necesario para el viaje de ida y vuelta de la madre para dar alimento a sus hijos. En este caso, el empleador pagará el valor de los pasajes por el transporte que deba emplearse para la ida y regreso de la madre. ${ }^{74}$

\footnotetext{
${ }^{74}$ Artículo sustituido, como aparece en el texto, por el Artículo Único № 2, de la Ley № 20.166 (D.O. 12.02.07).
} 